\title{
Study of rectangular waveguides with grooves of irregular shape using the finite element method
}

\author{
S K POPALGHAT ${ }^{1}$ and P B PATIL ${ }^{2, *}$ \\ ${ }^{1}$ Department of Physics, J. E. S. College, Jalna 431 203, India \\ ${ }^{2}$ Department of Physics, Dr Babasaheb Ambedkar Marathwada University, \\ Aurangabad 431004 , India
}

MS received 3 September 1997

\begin{abstract}
Electromagnetic wave propagation through grooved waveguides is studied using the finite element method (FEM). The effect of grooves of irregular shape on $\mathrm{TE}_{10}, \mathrm{TE}_{20}$ mode frequencies and passband is studied. The variation in cutoff frequencies for $\mathrm{TE}_{10}, \mathrm{TE}_{20}$ mode and passband is observed.
\end{abstract}

Keywords. Rectangular waveguides; grooves of irregular shape; finite element method; cutoff frequencies.

\section{Introduction}

A survey of the work on grooved waveguides indicates that a grooved waveguide has the advantages of low loss transmission, low dimensional tolerance and high power handling capacity at higher frequencies. It is also noted that these waveguides can effectively reject higher order modes.

The analysis of V-grooved waveguides of open and closed type has been done by various workers (Harris \& Lee 1981; Ho \& Harris 1984; Oliner \& Lampariello 1985; Choi et al 1988; Li et al 1991; Schutt Aine 1992) using different methods. Rectangular-grooved guides of various dimensions have also been studied (Sachidananda 1992) for cutoff wavelengths and various higher modes. The single V-grooved guide was studied by Li et al (1991) and Oliner \& Lampariello (1985) and propagation characteristics worked out for various dimensions, using the transverse resonance technique. Choi et al (1988) analysed single and double V-grooved waveguides by a conformal technique. Sachidananda (1992) used a mode matching technique to study the rectangular-grooved waveguide and reported the variation of cut-off wavelength by varying plate distance for constant groove width.

The work done on grooved guide as mentioned above is restricted to two definite shapes of grooves, V-shaped and rectangular. In this paper, rectangular waveguides with irregular

\footnotetext{
* Corresponding author
} 


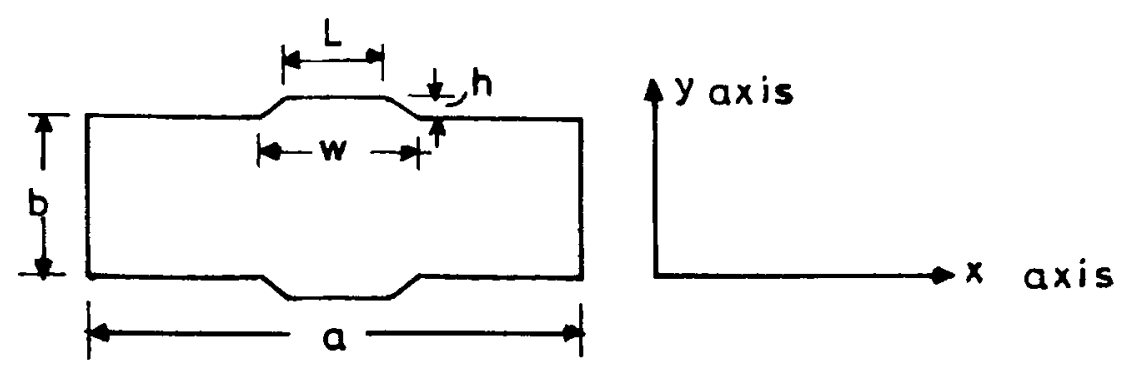

Figure 1. Cross-section of waveguide with symmetric groove of irregular shape.

grooves are studied by using the finite element method (Webb 1988; Dillon \& Webb 1994; Popalghat 1995). The effect of the irregularity of grooves on the cut-off frequency for the dominant mode $\mathrm{TE}_{10}$ and the mode next to it, i.e. $\mathrm{TE}_{20}$ is studied.

\section{Statement of the problem}

A homogeneous rectangular waveguide, with grooves of irregular shape at the centre of both its wide walls (as shown in figure 1), is considered. The walls of the waveguide are perfectly conducting, enclosing air as the dielectric medium.

The cross-section of the grooved waveguide under study is considered in the $X-Y$ plane of the Cartesian coordinate system as a problem domain $\Omega$ with conducting boundaries. The domain $\Omega$ under study has as its boundaries, electric walls (Webb 1988) on which normal magnetic components and tangential electric components vanish at all the boundary segments.

The electric and magnetic fields inside the waveguide satisfy Maxwell's equations. With time dependence $=\exp (j \omega t)$, Maxwell's curl equations can be combined to give

$$
\nabla \times\left(\epsilon_{r}^{-1} \nabla \times \mathbf{H}\right)=K_{0}^{2} \mathbf{H},
$$

where $K_{0}^{2}=\omega^{2} \mu_{0} \epsilon_{0}$.

Here $\omega$ is the angular frequency, $\epsilon_{0}$ the permittivity and $\mu_{0}$ the permeability of free space.

Table 1. Frequencies $(f)$ and bandwidths (BW) for grooved waveguides of irregular shape. $W=1.6 \mathrm{~cm}, h=0.15 \mathrm{~cm}$.

\begin{tabular}{lccc}
\hline$L / W$ & \multicolumn{2}{c}{$f(\mathrm{GHz})$} & $\begin{array}{c}\mathrm{BW} \\
(\mathrm{GHz})\end{array}$ \\
\cline { 2 - 3 } & $\mathrm{TE}_{10}$ & $\mathrm{TE}_{20}$ & 7.0467 \\
0.1250 & 5.9511 & 12.9977 & 7.0165 \\
0.2500 & 5.9123 & 12.9288 & 6.9567 \\
0.3750 & 5.8779 & 12.8346 & 6.8678 \\
0.5000 & 5.8487 & 12.7165 & 6.7611 \\
0.6250 & 5.8257 & 12.5868 & 6.6347 \\
0.7500 & 5.8086 & 12.4433 & 6.5139 \\
0.8750 & 5.7958 & 12.3097 & 6.2872 \\
1.1250 & 5.7754 & 12.0626 & 6.1764 \\
1.2500 & 5.7595 & 11.9360 & 6.0463 \\
1.3750 & 5.7484 & 11.7947 & \\
\hline
\end{tabular}


Table 2. Frequencies $(f)$ and bandwidths $(\mathrm{BW})$ for grooved waveguides of irregular shape. $W=1.6 \mathrm{~cm}, h=0.3 \mathrm{~cm}$.

\begin{tabular}{lccc}
\hline$L / W$ & \multicolumn{2}{c}{$f(\mathrm{GHz})$} & $\begin{array}{c}\mathrm{BW} \\
(\mathrm{GHz})\end{array}$ \\
\cline { 2 - 3 } & $\mathrm{TE}_{10}$ & $\mathrm{TE}_{20}$ & 7.3048 \\
0.1250 & 5.5951 & 12.8999 & 7.3194 \\
0.2500 & 5.5314 & 12.8508 & 7.3317 \\
0.5000 & 5.4759 & 12.8075 & 7.2009 \\
0.6250 & 5.4274 & 12.6283 & 7.0615 \\
0.7500 & 5.3846 & 12.4461 & 6.8781 \\
0.8750 & 5.3463 & 12.2244 & 6.6645 \\
1.1250 & 5.3108 & 11.9753 & 6.1860 \\
1.2500 & 5.2460 & 11.4321 & 5.9282 \\
1.3750 & 5.2147 & 11.1428 & 5.6538 \\
\hline
\end{tabular}

The magnetic field $\mathbf{H}$ must satisfy the appropriate boundary condition at the conducting boundaries, the condition being that the tangential component of the electric field be zero at the conductor boundaries,

$$
\mathbf{n} \times \nabla \times\left.\mathbf{H}\right|_{\text {boundary }}=0 .
$$

\section{FEM formulation}

The expression for functional $\Pi$ is

$$
\Pi=\frac{1}{2} \int_{\Omega}\left[\left(\nabla \times \mathbf{H}^{*}\right) \cdot\left(\epsilon_{r}^{-1} \nabla \times \mathbf{H}\right)-K_{0}^{2} \mathbf{H}^{*} \cdot \mathbf{H}+\left(\nabla \cdot \mathbf{H}^{*}\right)(\nabla \cdot \mathbf{H})\right] \mathrm{d} \Omega .
$$

The stationary character of $\Pi$ requires $\delta \Pi=0$.

The first variation in $\Pi$ is given by,

$$
\delta\left[\int_{\Omega}\left(\nabla \times \mathbf{H}^{*}\right) \cdot\left(\epsilon_{r}^{-1} \nabla \times \mathbf{H}\right) \mathrm{d} \Omega-\int_{\Omega} K_{0}^{2} \mathbf{H}^{*} \cdot \mathbf{H} \mathrm{d} \Omega+\int_{\Omega}\left(\nabla \cdot \mathbf{H}^{*}\right)(\nabla \cdot \mathbf{H}) \mathrm{d} \Omega\right] .
$$

Table 3. Frequencies $(f)$ and bandwidths (BW) for grooved waveguides of irregular shape. $W=1.2 \mathrm{~cm}, h=0.15 \mathrm{~cm}$.

\begin{tabular}{lccc}
\hline$L / W$ & \multicolumn{2}{c}{$f(\mathrm{GHz})$} & $\begin{array}{c}\mathrm{BW} \\
(\mathrm{GHz})\end{array}$ \\
\cline { 2 - 3 } & $\mathrm{TE}_{10}$ & $\mathrm{TE}_{20}$ & 6.9861 \\
0.1667 & 5.9438 & 12.9299 & 7.0269 \\
0.3334 & 5.8990 & 12.9259 & 7.0383 \\
0.5000 & 5.8582 & 12.8965 & 7.0157 \\
0.6667 & 5.8225 & 12.8352 & 6.9607 \\
0.8334 & 5.7911 & 12.7518 & 6.7860 \\
1.1667 & 5.7336 & 12.5195 & 6.6758 \\
1.3334 & 5.7053 & 12.3811 & 6.5417 \\
1.5000 & 5.6774 & 12.2191 & 6.3701 \\
1.6667 & 5.6496 & 12.0197 & 6.1469 \\
1.8334 & 5.6215 & 11.7684 & \\
\hline
\end{tabular}


Table 4. Frequencies $(f)$ and bandwidths (BW) for grooved waveguides of irregular shape. $W=1.2 \mathrm{~cm}, h=0.3 \mathrm{~cm}$.

\begin{tabular}{lccc}
\hline$L / W$ & \multicolumn{2}{c}{$f(\mathrm{GHz})$} & $\begin{array}{c}\mathrm{B} \mathrm{W} \\
(\mathrm{GHz})\end{array}$ \\
\cline { 2 - 3 } & $\mathrm{TE}_{10}$ & $\mathrm{TE}_{20}$ & 7.4069 \\
0.1667 & 5.6185 & 13.0254 & 7.4892 \\
0.3334 & 5.5394 & 13.0286 & 7.5362 \\
0.5000 & 5.4675 & 13.0037 & 7.5372 \\
0.6667 & 5.4013 & 12.9384 & 7.4819 \\
0.8334 & 5.3395 & 12.8214 & 7.1744 \\
1.1667 & 5.2258 & 12.4002 & 6.9245 \\
1.3334 & 5.1716 & 12.0962 & 6.6182 \\
1.5000 & 5.1180 & 11.7362 & 6.2601 \\
1.6667 & 5.0643 & 11.3244 & 5.8569 \\
1.8334 & 5.0104 & 10.8673 & \\
\hline
\end{tabular}

The domain (i.e. cross-section of the waveguide) is divided into rectangular elements with four nodes. The functional over each element is,

$$
\begin{aligned}
\Pi^{e}= & \sum \frac{1}{2}\left[\int_{\Omega^{e}}\left(\nabla \times H^{e *}\right) \cdot\left(\epsilon_{r}^{-1} \nabla \times H^{e}\right) \mathrm{d} \Omega^{e}\right. \\
& \left.-\int_{\Omega^{e}} K_{0}^{2} H^{e *} \cdot H^{e} \mathrm{~d} \Omega^{e}+\int_{\Omega^{e}}\left(\nabla \cdot H^{e *}\right)\left(\nabla \cdot H^{e}\right) \mathrm{d} \Omega^{e}\right],
\end{aligned}
$$

where the unknown vector $H^{e}$ has three components $H x, H y, H z$. Each unknown component $H x, H y, H z$ is defined in terms of the nodal values $H_{x}^{e}, H_{y}^{e}, H_{z}^{e}$ respectively.

Linear mapping functions are used for 4 nodes per rectangular element.

Functional $\Pi^{e}$ can be written as,

$$
\Pi^{e}=\sum \frac{1}{2}\left\{H^{e}\right\}^{\mathrm{T}}\left(\left[S^{\mathrm{e}}\right]-K_{0}^{2}\left[T^{e}\right]\right)\left\{H^{e}\right\} .
$$

The functional for the whole region $\Omega$ is given by,

$$
\Pi=\frac{1}{2}\{H\}^{T}[S]\{H\}-\frac{1}{2} K_{0}^{2}\{H\}^{T}[T]\{H\} .
$$

The condition $\delta \pi / \delta\{H\}=0$ leads to the following matrix equation

$$
[S]\{H\}-K_{0}^{2}[T]\{H\}=0 .
$$

Table 5. Frequencies $(f)$ and bandwidths (BW) for grooved waveguides of irregular shape. $W=0.8 \mathrm{~cm}, h=0.15 \mathrm{~cm}$.

\begin{tabular}{lccc}
\hline$L / W$ & \multicolumn{2}{c}{$f(\mathrm{GHz})$} & $\mathrm{B} W$ \\
& $\mathrm{TE}_{10}$ & $\mathrm{TE}_{20}$ & $(\mathrm{GHz})$ \\
\hline 0.2500 & 5.9889 & 12.9377 & 6.9488 \\
0.5000 & 5.9367 & 12.9673 & 7.0306 \\
0.7500 & 5.8879 & 12.9756 & 7.0877 \\
1.2500 & 5.7972 & 12.9182 & 7.1210 \\
1.5000 & 5.7530 & 12.8577 & 7.1047 \\
1.7500 & 5.7089 & 12.7733 & 7.0645 \\
2.0000 & 5.6644 & 12.6498 & 6.9855 \\
2.2500 & 5.6195 & 12.4600 & 6.8405 \\
2.5000 & 5.5737 & 12.1686 & 6.5949 \\
2.7500 & 5.5271 & 11.7499 & 6.2228 \\
\hline
\end{tabular}


Table 6. Frequencies $(f)$ and bandwidths (BW) for grooved waveguides of irregular shape. $W=0.8 \mathrm{~cm}, h=0.3 \mathrm{~cm}$.

\begin{tabular}{lccc}
\hline$L / W$ & \multicolumn{2}{c}{$f(\mathrm{GHz})$} & $\begin{array}{c}\mathrm{B} \mathrm{W} \\
(\mathrm{GHz})\end{array}$ \\
\cline { 2 - 3 } & $\mathrm{TE}_{10}$ & $\mathrm{TE}_{20}$ & 7.2844 \\
0.2500 & 5.7160 & 13.0005 & 7.4010 \\
0.5000 & 5.6175 & 13.0184 & 7.5071 \\
1.2500 & 5.5252 & 13.0323 & 7.6637 \\
1.5000 & 5.3544 & 13.0181 & 7.6878 \\
1.7500 & 5.2740 & 12.9618 & 7.6439 \\
2.0000 & 5.1954 & 12.8393 & 7.4833 \\
2.2500 & 5.1182 & 12.6015 & 7.1459 \\
2.5000 & 5.0417 & 12.1876 & 6.6294 \\
2.7500 & 4.9659 & 11.5952 & 6.0202 \\
\hline
\end{tabular}

\section{Numerical calculations}

Consider an irregular groove on the wide wall of an $X$-band rectangular waveguide of cross-sectional length $a=2.4 \mathrm{~cm}$ and breadth $b=1.2 \mathrm{~cm}$. The groove is symmetric. By keeping base width $W$ and height $h$ of the groove fixed, the inclination of the grooved side is varied by varying the groove top length $L$, from $0.2 \mathrm{~cm}$ to $2.2 \mathrm{~cm}$, in steps of $0.2 \mathrm{~cm}$.

The analysis is repeated for three different base widths $W=1.6,1.2$, and $0.8 \mathrm{~cm}$, and for two different heights $h=0.15$ and $0.3 \mathrm{~cm}$. The results are presented in tables 1-6.

For each $W$ and for two different $h, L$ is varied and the effect of this on $\mathrm{TE}_{10}$ and $\mathrm{TE}_{20}$ mode frequencies and bandwidth is studied. The variation of $\mathrm{TE}_{10}$ and $\mathrm{TE}_{20}$ are shown

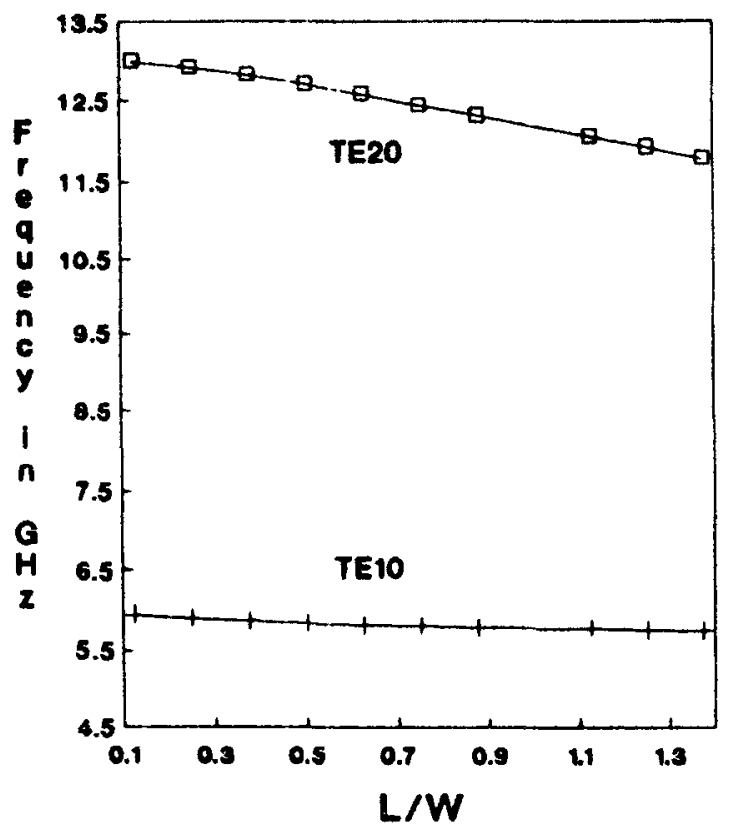

Figure 2. Variation of $\mathrm{TE}_{10}$ and $\mathrm{TE}_{20}$ mode frequencies for waveguides with grooves of irregular shape with $W=1.6 \mathrm{~cm}$ and $h=$ $0.15 \mathrm{~cm}$, for different $L / W$ ratios. 


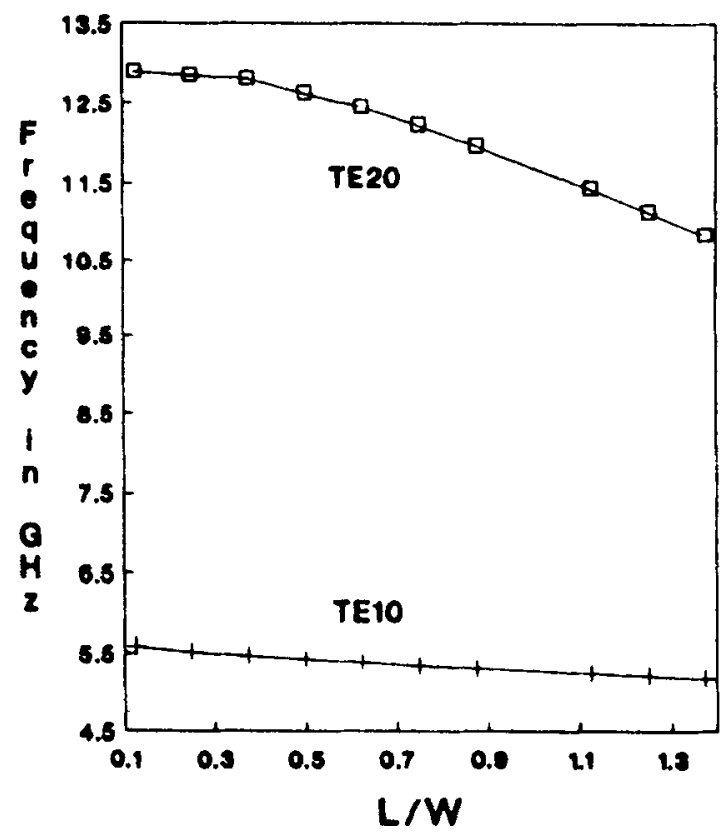

Figure 3. Variation of $\mathrm{TE}_{10}$ and $\mathrm{TE}_{20}$ mode frequencies for waveguides as in figure 2 , with $W=1.6 \mathrm{~cm}$ and $h=0.3 \mathrm{~cm}$, for different $L / W$ ratios.

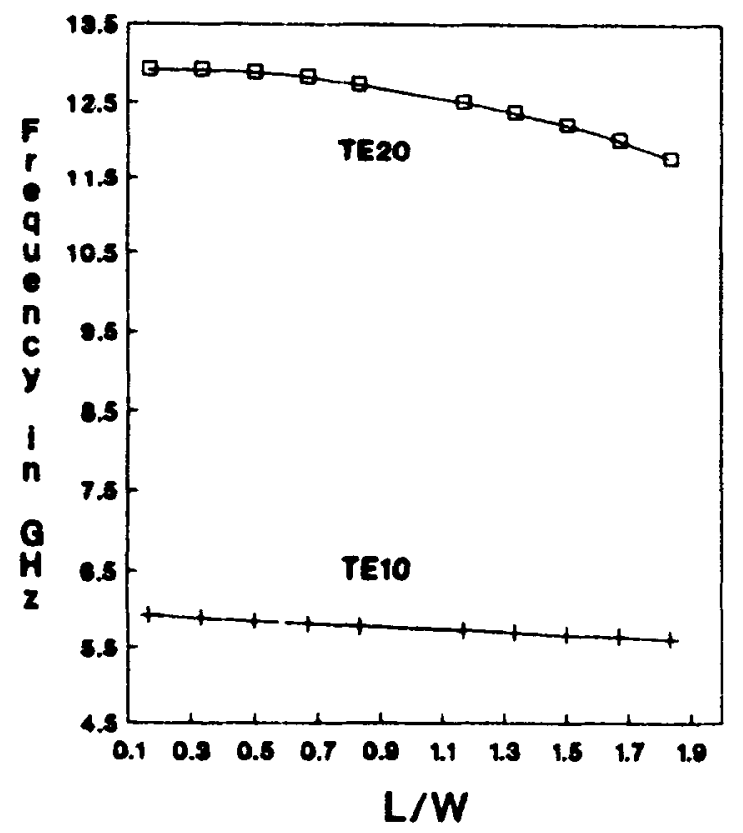

Figure 4. Variation of $\mathrm{TE}_{10}$ and $\mathrm{TE}_{20}$ mode frequencies for waveguides as in figure 2 , with $W=1.2 \mathrm{~cm}$ and $h=0.15 \mathrm{~cm}$ for different $L / W$ ratios. 

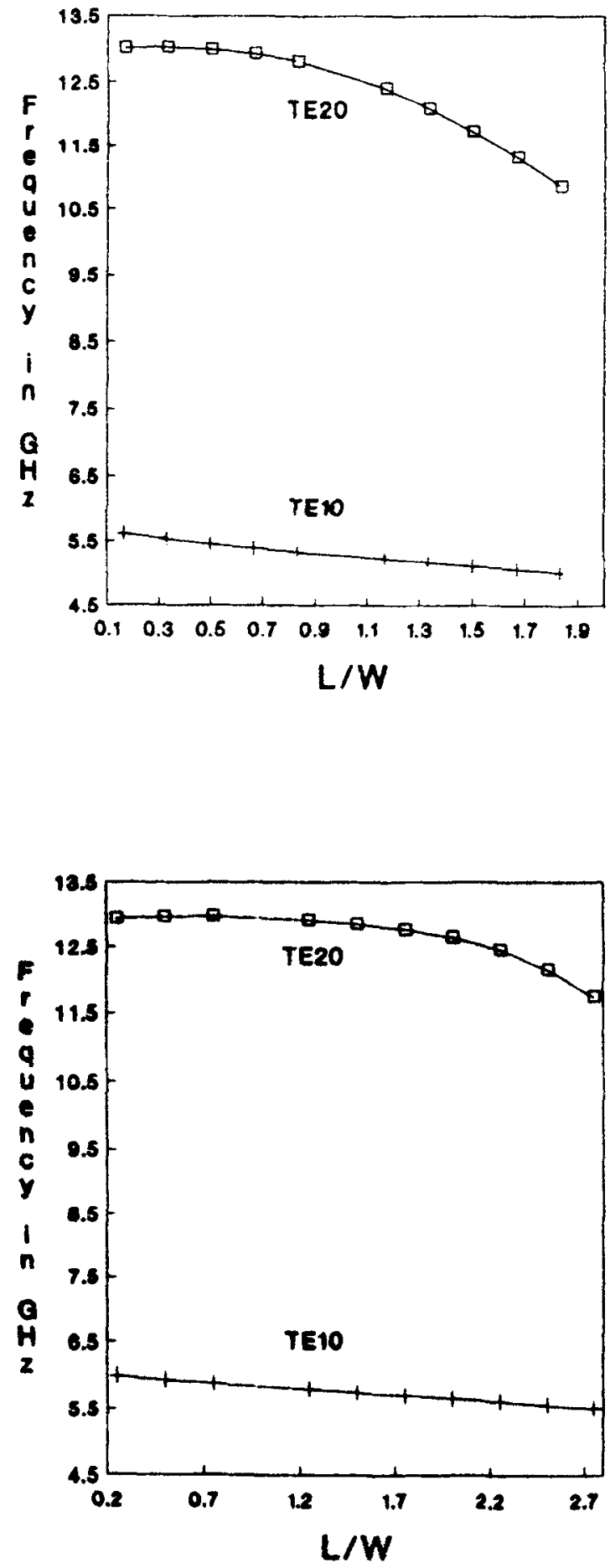

Figure 5. Variation of $\mathrm{TE}_{10}$ and $\mathrm{TE}_{20}$ mode frequencies waveguides as in figure 2 , with $W=1.2 \mathrm{~cm}$ and $h=0.3 \mathrm{~cm}$, for different $L / W$ ratios.

Figure 6. Variation of $\mathrm{TE}_{10}$ and $\mathrm{TE}_{20}$ mode frequencies for waveguides as in figure 2 , with $W=0.8 \mathrm{~cm}$ and $h=0.15 \mathrm{~cm}$, for different $L / W$ ratios. 

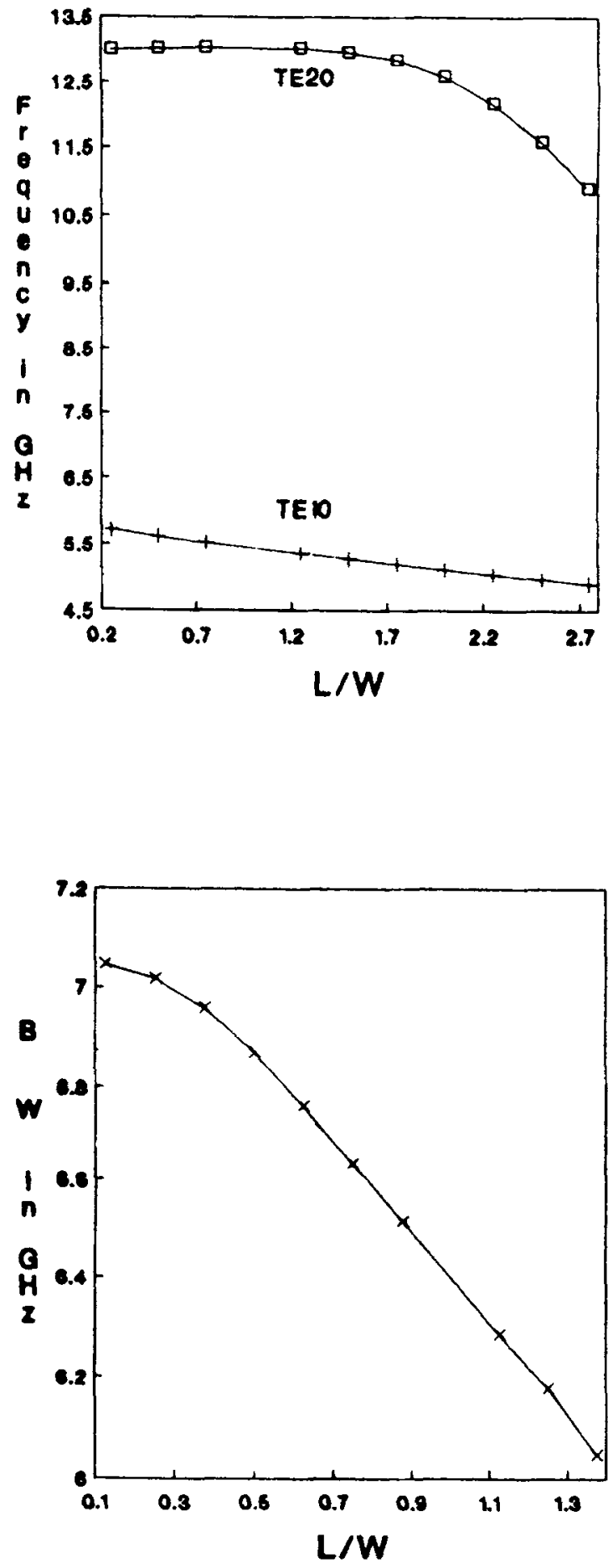

Figure 7. Variation of $\mathrm{TE}_{10}$ and $\mathrm{TE}_{20}$ mode frequencies for waveguides as in figure 2 , with $W=0.8 \mathrm{~cm}$ and $h=0.3 \mathrm{~cm}$ for different $L / W$ ratios.

Figure 8. Variation of bandwidth for waveguides as in figure 2 , with $W=1.6 \mathrm{~cm}$ and $h=0.15 \mathrm{~cm}$, for different ratios of $L / W$. 

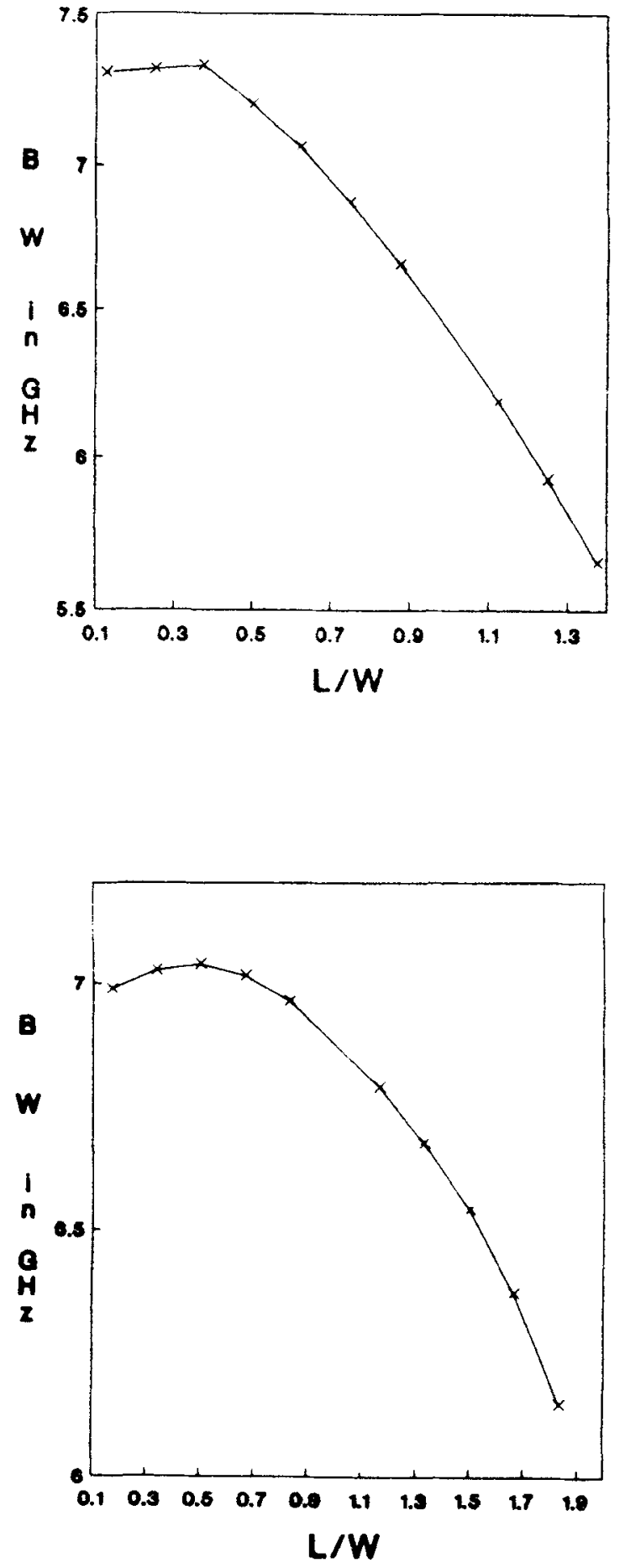

Figure 9. Variation of bandwidth for waveguides as in figure $2, W=1.6 \mathrm{~cm}$ and $h=$ $0.3 \mathrm{~cm}$, for different ratios of $L / W$.

Figure 10. Variation of bandwidth for waveguides as in figure 2 , with $W=1.2 \mathrm{~cm}$ and $h=0.15 \mathrm{~cm}$, for different ratios of $L / W$. 

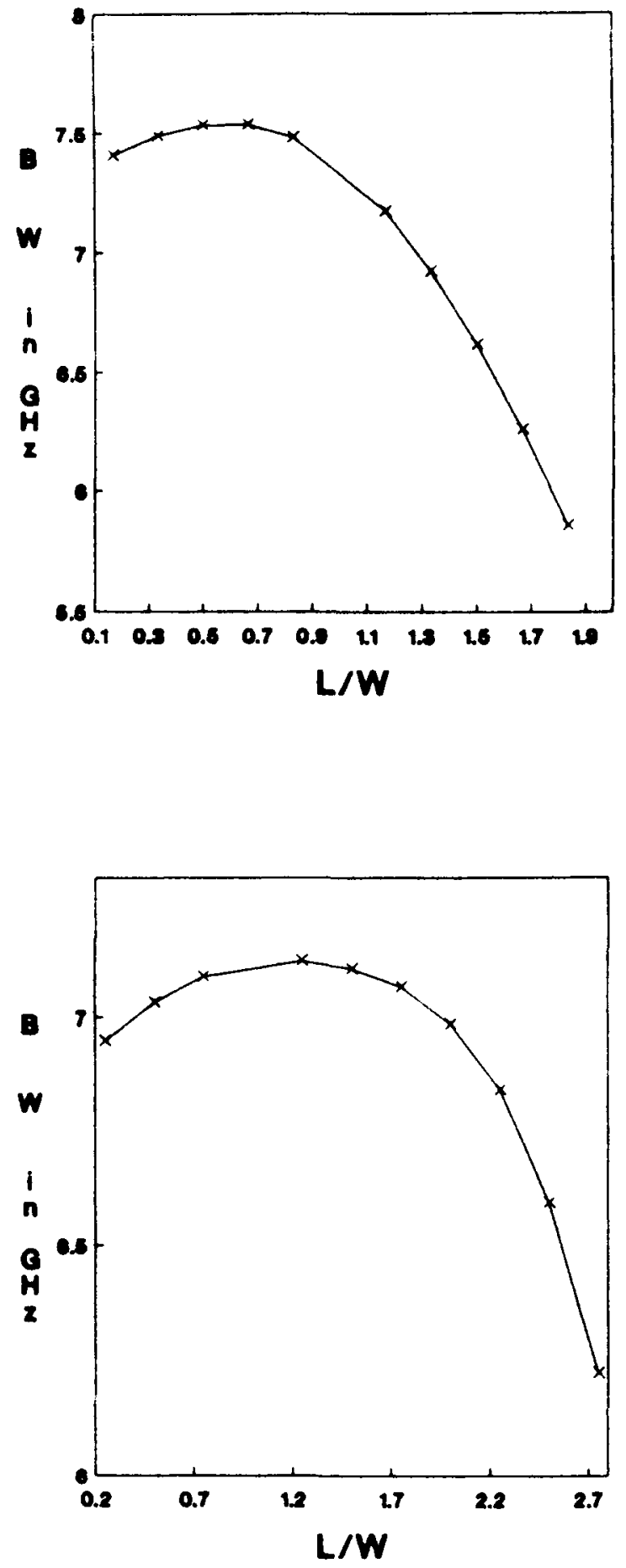

Figure 11. Variation of bandwidth for waveguide as in figure 2 , with $W=1.2 \mathrm{~cm}$ and $h=0.3 \mathrm{~cm}$, for different ratios of $L / W$.

Figure 12. Variation of bandwidth for waveguides as in figure 2 , with $W=0.8 \mathrm{~cm}$ and $h=0.15 \mathrm{~cm}$, for different ratios of $L / W$. 


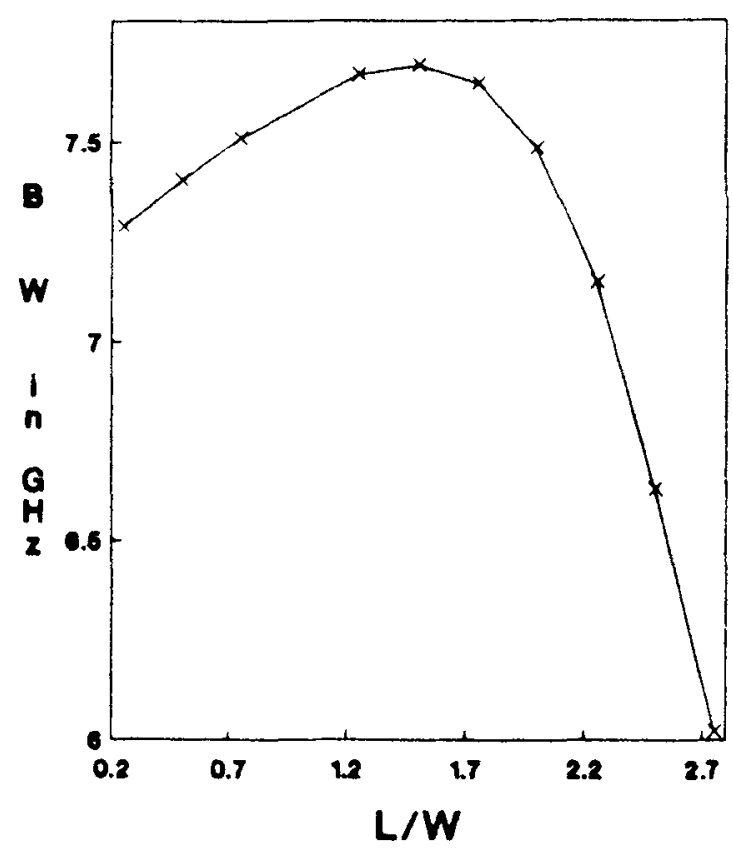

Figure 13. Variation of bandwidth for waveguides as in figure 2 , with $W=0.8 \mathrm{~cm}$ and $h=0.3 \mathrm{~cm}$, for different ratios of $L / W$.

in figures 2 to 7 . The variations of bandwidth with ratio $L / W$ for each case are shown in figures 8 to 13 .

\section{Conclusions}

Bandwidth is higher than that of a normal waveguide when $L / W<1$ and it decreases as $L / W$ increases. When $L / W>1$, the bandwidth is less than that of the normal waveguide and decreases further with increase in $L / W$. For a specific value of $L / W$, the bandwidth is higher for greater groove height.

Bandwidth variations can be helpful for using waveguides as pass-band components whose pass-band range can be widened or suppressed by proper choice of groove height and suitable $L / W$ ratios.

Financial support from the University Grants Commission, New Delhi is gratefully acknowledged.

\section{References}

Choi Y M, Harris D J, Tsang K F 1988 Theoretical and experimental characteristics of single $\mathrm{V}$-groove guide for $X$-band and $100 \mathrm{GHz}$ operation. IEEE Trans. Microwave Theory Tech. 36: $715-723$ 
Dillon B M, Webb J P 1994 A comparison of formulations for the vector finite element analysis of waveguides. IEEE Trans. Microwave Theory Tech. 42: 308-316

Harris D J, Lee K W 1981 Theoretical and experimental characteristics of double-groove guide for $100 \mathrm{GHz}$ operation. Proc. Inst. Elect. Eng. H128: 6-10

Ho T K, Harris D J 1984 Millimeter wave groove guide with V-shaped grooves. Electron. Lett. 20: $777-778$

Li S-F, Shen Z-X, Lou X-M 1991 A simple analysis of single and double V-groove guides. IEEE Trans. Microwave Theory Tech. 39: 1413-1416

Oliner A A, Lampariello P 1985 The combinant mode properties of open goove guide; an improved solution. IEEE Trans. Microwave Theory Tech. 33: 755--764

Popalghat S K 1995 Study of electromagnetic propagation using finite element method. Ph D thesis, Dr Babasaheb Ambedkar Marathwada University, Aurangabad

Sachidananda I M 1992 Rigorous analysis of a groove waveguide. IEEE Proc. H139: 449-452

Schutt Aine J E 1992 Static analysis of V transmission lines. IEEE Trans. Microwave Theory Tech. 40: 654-664

Webb J P 1988 Finite element analysis of dispersion in waveguides with sharp metal edges. IEEE Trans. Microwave Theory Tech. 36: 1819-1824 